\title{
FUNDAÇÕES DE CONSTRUÇÕES SUBMETIDAS A ESFORÇOS DE TRAÇÃO EM SOLO DE ALTA POROSIDADE DA REGIÃO DE CAMPINAS - SP
}

\section{JOÃO A. PASCHOALIN FILHO ${ }^{1}$, DAVID DE CARVALHO ${ }^{2}$}

\begin{abstract}
RESUMO: Considerando-se que a maior parte de construções rurais utiliza fundações por estacas escavadas, pequisou-se, neste trabalho, o comportamento à tração de três estacas, submetidas a esforços verticais de tração. $\mathrm{O}$ comportamento à tração foi pesquisado, por ser esta situação bastante comum em estruturas leves, como silos, galpões, caixas d'água elevadas, etc. As estacas foram executadas no Campo Experimental para Estudos de Mecânica dos Solos e Fundações da Unicamp, localizado na cidade de Campinas - SP. O subsolo do local é constituído por um solo poroso de Diabásio, cuja primeira camada de 6,5 m é constituída de argila silto-arenosa, seguida de uma camada de silte argilo-arenoso e nível do lençol freático a $17 \mathrm{~m}$ de profundidade. Para a verificação do comportamento destas estacas, foram executadas provas de carga de acordo com a NBR 12131. A capacidade de carga destas estacas foi prevista por meio da utilização de métodos teóricos próprios para esforços de tração e semiempirícos (próprios para esforços de compressão), considerando neste último somente a parcela de resistência lateral e superfície de ruptura no contato estaca/solo.
\end{abstract}

PALAVRAS-CHAVE: construções rurais, estacas escavadas, esforços de tração.

\section{RURAL CONSTRUCTION FOUNDATIONS SUBMITTED TO UPLIFT EFFORTS IN HIGH POROSITY SOIL OF THE REGION OF CAMPINAS - SP, BRAZIL}

\begin{abstract}
This paper presents the behavior of three bored piles conducted in diabasic soil submitted to uplift forces. The piles were built at the site for Experimental Studies in Soil Mechanics and Foundations of UNICAMP, located in the city of Campinas, Brazil. Field tests have already been conducted at the site (SPT, CPT, DMT and PMT), as well as laboratory tests by using sample soils taken from a well up to $17 \mathrm{~m}$ deep. The water table is not checked until a depth of $17 \mathrm{~m}$. In order to check the behavior of the piles when submitted to uplift forces, slow static load tests were carried out as the recommendations of NBR 12131. The carrying capacity of these piles was also provided by means of theoretical methods, appropriate for uplift forces, and through semiempirical methods appropriate for compression forces, considering only the portion of lateral resistance. The values estimated by using the considered methods were compared to those obtained by means of load tests. One of the tested piles was extracted from the soil to be the subject of a study on its geometry.
\end{abstract}

KEYWORDS: rural constructions, bored piles, uplift efforts.

\section{INTRODUÇÃO}

Fundações tracionadas podem ser encontradas com facilidade em obras rurais. Pode-se citar como exemplo: fundações de galpões metálicos, torres de transmissão de energia elétrica e silos verticais.

Estas construções são caracterizadas por serem altamente sujeitas a esforços de vento, que, aliado ao baixo peso próprio destas estruturas, poderá induzir ao bloco de fundação momentos que tenderão a tracionar as fundações.

\footnotetext{
${ }^{1}$ Prof. Dr., Universidade Nove de Julho - Engenharias, Coordenador Adjunto Engenharias, Fone: (0XX11) 3665.9028, jalexandre@uninove.br.

${ }^{2}$ Professor Associado, FEAGRI, UNICAMP, david@ feagri.unicamp.br.

Recebido pelo Conselho Editorial em: 28-9-2008

Aprovado pelo Conselho Editorial em: 4-11-2009
} 
Dentre os tipos de estacas utilizadas em obras rurais, destacam-se as do tipo escavada em função de facilidade executiva e baixos custos.

O processo executivo destas consiste na perfuração do terreno, utilizando-se de um trado helicoidal mecânico ou manual. A escavação manual, apesar de ser mais barata, apresenta como desvantagens principais a limitação do diâmetro máximo da estaca e sua profundidade de implantação. Por outro lado, o processo mecânico permite ao engenheiro projetar estacas com diâmetros maiores e utilizar maiores profundidades, entretanto o custo é mais elevado. Após a perfuração, é instalada a armadura e, posteriormente, lança-se o concreto.

A carga de ruptura de uma fundação tracionada pode ser quantificada como sendo o peso próprio do elemento de fundação, somado ao peso de uma massa de solo contida no interior da superfície de ruptura e a resistência por cisalhamento, que ocorre nesta superfície de ruptura.

Este problema nem sempre é de fácil resolução, pois a quantificação do peso do solo deverá ser função do formato da superfície de ruptura, o que ainda representa um ponto de discussão importante entre os projetistas de fundações. Outra dificuldade consiste na determinação dos parâmetros de resistência ao cisalhamento desta superfície, pois estes dependerão tanto de características geomecânicas do solo como do tipo e da maneira de execução da fundação (PASCHOALIN FILHO et al., 2006).

Diante dessa situação, é prática comum entre diversos projetistas a adoção de métodos empíricos e semiempíricos desenvolvidos para estacas submetidas a esforços axiais de compressão. Adota-se, neste caso, a hipótese de que a resistência última de uma estaca tracionada corresponderia uma porcentagem da resistência lateral no instante de ruptura de uma mesma estaca (com iguais dimensões) solicitada a esforços compressivos. Ressalta-se que, ainda, não existe um valor comum entre os projetistas (PASCHOALIN FILHO et al., 2008).

Esta prática é puramente empírica e não leva em consideração os mecanismos de ruptura à tração típicos de cada estaca. Tal suposição também poderá acarretar em erros, pois geralmente um determinado método que se apresenta como adequado para uma determinada condição, em outra poderá fornecer parâmetros de previsão de capacidade de carga à tração totalmente fora do real, além do fato de algumas equações, empíricas ou semiempíricas, apresentarem grande grau de dispersão de seus resultados, mesmo quando são utilizadas para a previsão de capacidade de carga de estacas comprimidas (PASCHOALIN FILHO, 2008).

\section{MATERIAL E MÉTODOS}

O Campo Experimental Feagri/Unicamp está localizado na Universidade Estadual de Campinas, no município de Campinas - SP, e localiza-se na porção centro-leste do Estado de São Paulo, no Planalto Atlântico. Sua posição geográfica é determinada pelas coordenadas $22^{\circ} 53^{\prime} 22^{\prime}$ ' de latitude S e 4704'39', de longitude W.

No local, já foram executados diversos tipos de ensaios de campo, além de provas de carga estáticas do tipo lenta e rápida (com solicitações à compressão, tração e horizontal), em estacas executadas in loco, além de ensaios de carregamento dinâmico em estacas pré-moldadas de concreto e estacas metálicas. Na Tabela 1, apresentam-se alguns parâmetros geotécnicos do campo em estudo.

TABELA 1. Parâmetros geotécnicos médios. Average geotechnical parameters.

\begin{tabular}{c|c|c|c|c}
\hline Profundidade & pnat $\left(\mathrm{kN} \mathrm{m}^{-3}\right)$ & $\mathrm{n}(\%)$ & $\mathrm{c}^{*}(\mathrm{kPa})$ & $\emptyset^{*}\left({ }^{\circ}\right)$ \\
\hline $1-3 \mathrm{~m}$ & 13,0 & 64,0 & 9,0 & 31,5 \\
$4-8 \mathrm{~m}$ & 14,5 & 25,0 & 17,0 & 22,1 \\
$9-12 \mathrm{~m}$ & 15,5 & 31,0 & 76,3 & 18,5 \\
$13-16 \mathrm{~m}$ & 16,5 & 35,0 & 65,5 & 20,5 \\
\hline
\end{tabular}

* Valores em termos de tensões totais. pnat - peso específico natural; $\mathrm{n}$ - porosidade; c - coesão; $\varnothing$ - ângulo de atrito. 
O subsolo da região do campo experimental é formado por magmatitos básicos, sendo observada a presença de rochas intrusivas básicas da formação Serra Geral. Este subsolo ocupa um total de $98 \mathrm{~km}^{2}$, correspondendo a $14 \%$ de sua área total do município de Campinas (ALBUQUERQUE, 2001).

Em termos pedológicos, o solo presente na região pode ser classificado como Latossolo Vermelho eutroférrico, sendo composto por: quartzo, ilmenita, magnetita, caulinita, gibsita e hidróxidos de ferro.

O subsolo do Campo Experimental é formado por um horizonte superficial poroso, originado por meio da intemperização de Diabásio, de 6,5 m de espessura, constituído de argila silto-arenosa de alta porosidade. Subsequente a este horizonte, verifica-se uma camada de silte argilo-arenoso até 19 m; o nível d' água é encontrado a 17 m.

Na Figura 1, apresenta-se a variação dos valores de $\mathrm{N}_{\mathrm{spt}}$ com a profundidade.

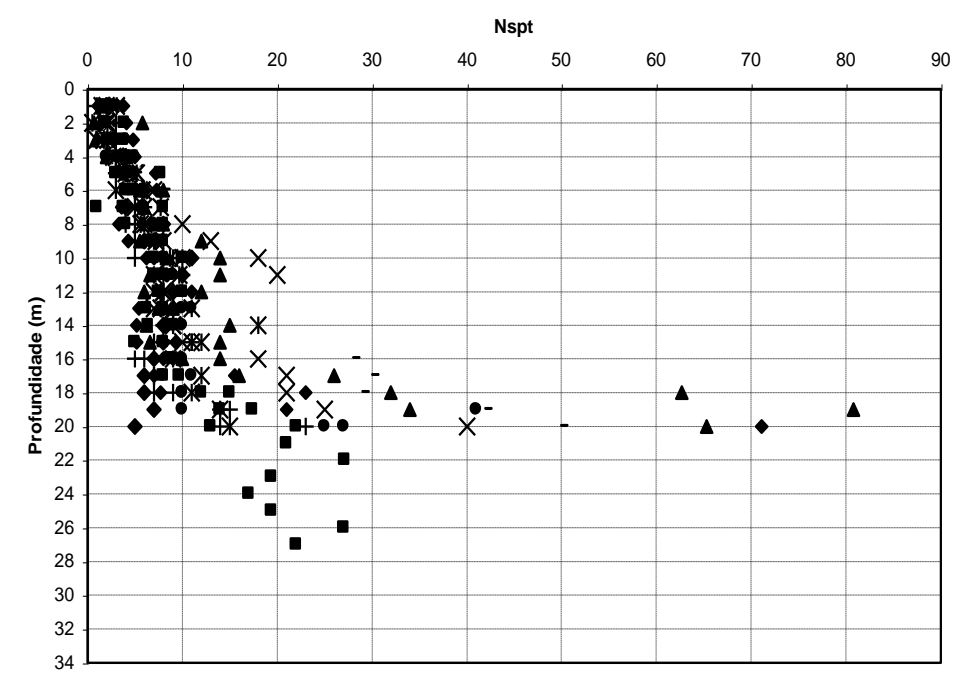

FIGURA 1. Variação dos valores de $\mathrm{N}_{\text {spt }}$ com a profundidade. Variation of $\mathbf{N}_{\text {spt }}$ values according to the depth.

Para esta pesquisa, foram implantadas três estacas escavadas com $12 \mathrm{~m}$ de profundidade e $400 \mathrm{~mm}$ de diâmetro. A operação de instalação destas seguiu rigorosamente os procedimentos recomendados pela ABEF (1999). A armadura longitudinal das estacas foi composta por quatro barras de aço do tipo CA-50 de $16 \mathrm{~mm}$ de diâmetro e estribos de $6,3 \mathrm{~mm}$ de diâmetro espaçados a cada $20 \mathrm{~cm}$. O valor do $f c k$ do concreto utilizado foi de $15 \mathrm{MPa}$, apresentando slump de $\pm 70 \mathrm{~mm}$.

Foram executadas provas de carga estáticas do tipo lenta, de acordo com as recomendações da NBR 12131 (ABNT, 1991). O sistema de carregamento consistiu na utilização de um macaco hidráulico (com capacidade para $2.000 \mathrm{kN}$ ) e uma célula de carga, sendo estes montados sobre uma viga metálica projetada para suportar esforços de até $1.500 \mathrm{kN}$ em sua parte central. Esta viga é constituída por um perfil duplo "I", tendo um comprimento de 5,30 m, altura de 0,75 m e largura de $0,80 \mathrm{~m}$ e peso total equivalente a $35 \mathrm{kN}$. Para a observação dos recalques decorrentes em cada estágio de carregamento, foram utilizados deflectômetros com precisão de leitura de $0,01 \mathrm{~mm}$ e curso de $50 \mathrm{~mm}$.

A montagem da prova de carga pode ser visualizada por meio da observação da Figura 2.

As cargas máximas foram previstas por meio da utilização de métodos teóricos, próprios para estacas tracionadas, e também por meio de métodos semiempíricos, próprios para estacas comprimidas. Os resultados obtidos por meio da aplicação dos métodos de previsão estudados foram comparados com os valores de carga de máxima obtidos nas provas de carga executadas. 


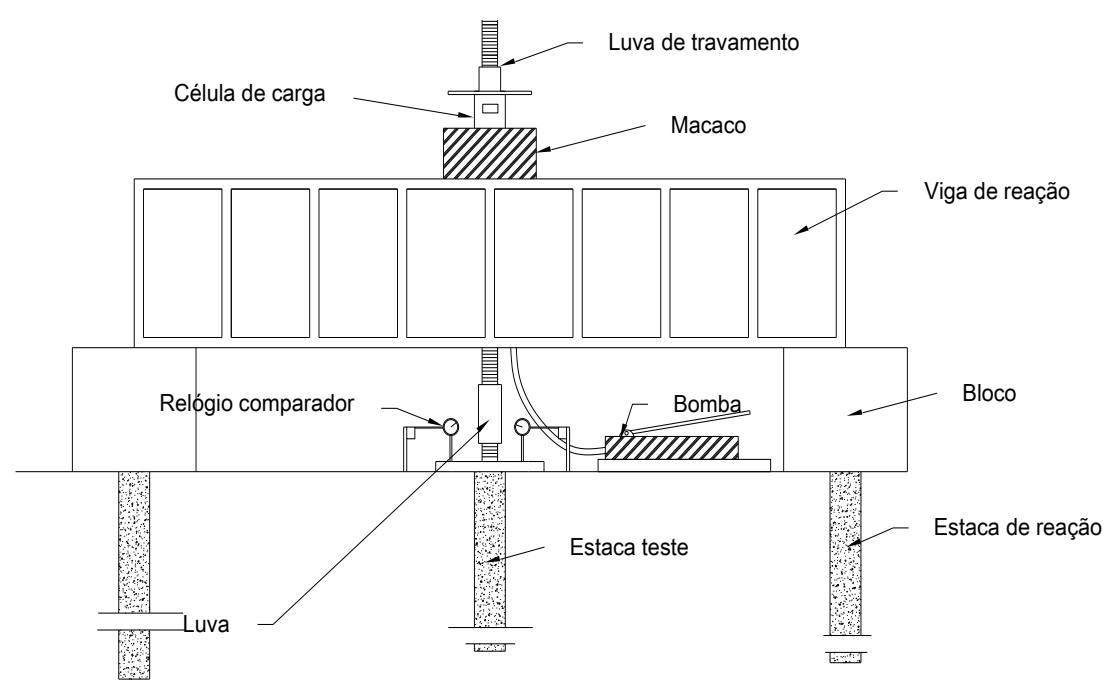

FIGURA 2. Croqui executivo da prova de carga. Static load test execution.

Os métodos teóricos contemplados nesta pesquisa foram: MEYERHOFF (1973) e da Universidade de Grenoble (BIARREZ \& BARRAUD, 1968 e MARTIN, 1966, 1973). Para a aplicação do método de MEYERHOFF (1973) foram adotados valores de ca $=0,80 \mathrm{c} ; \delta=0,95 \varnothing$, conforme POTYONDY (1961) e ca $=\mathrm{c}$ e $\delta=\emptyset$. Para a previsão das cargas máximas por meio do método da Universidade de Grenoble, foram adotados os valores de $\lambda=0$ e $\lambda=-\varnothing / 8$.

Os métodos semiempíricos contemplados nesta pesquisa foram os de DÉCOURT \& QUARESMA (1998) e AOKI \& VELLOSO (1975), utilizando valores obtidos por meio de ensaios tipo SPT e CPT (Cone Penetration Test) e admitindo-se que a superfície de cisalhamento na ruptura estaca-solo ocorreu no contato estaca-solo.

Estes métodos de previsão de carga de ruptura utilizados podem ser encontrados em detalhes em PASCHOALIN FILHO (2008).

\section{RESULTADOS E DISCUSSÃO}

$\mathrm{Na}$ Tabela 2, são apresentados os valores de carga e deslocamento máximos obtidos. $\mathrm{Na}$ Figura 3, apresentam-se as curvas de carga versus recalque, obtidas por meio das provas de carga.

As provas de carga executadas para as estacas escavadas foram levadas até a carga máxima de $600 \mathrm{kN}$, valor este definido pela capacidade do tirante de aço utilizado no sistema de reação da prova. Dessa maneira, houve necessidade de se estimarem as cargas de máximas para cada estaca, utilizando-se do método de VAN der VEEN (1953). Os valores estimados foram: $700 \mathrm{kN}$ e $630 \mathrm{kN}$ para as estacas EC01 e EC03, respectivamente. A diferença entre as cargas máximas obtidas pelas provas de carga executadas nas estacas EC01 e EC03, e as cargas de rupturas estimadas foram de $14,3 \%$ e $4,8 \%$, respectivamente.

TABELA 2. Valores de cargas e deslocamentos máximos obtidos. Values of maximum displacements and loads obtained by the load tests.

\begin{tabular}{lccccc}
\hline Estaca & $\begin{array}{c}\text { Comprimento } \\
(\mathrm{m})\end{array}$ & $\begin{array}{c}\text { Diâmetro } \\
(\mathrm{mm})\end{array}$ & $\begin{array}{c}\text { Carga Máxima } \\
\text { da Prova de } \\
\text { Carga (PC) }\end{array}$ & $\begin{array}{c}\text { Deslocamento } \\
\text { Máximo (mm) }\end{array}$ & $\begin{array}{c}\text { Deslocamento } \\
\text { para 50\% de PC }\end{array}$ \\
\hline Escavada EC01 & 12 & 400 & $600 \mathrm{kN}$ & 16,62 & 4,92 \\
Escavada EC02 & 12 & 400 & $600 \mathrm{kN}$ & 41,48 & 4,46 \\
Escavada EC03 & 12 & 400 & $600 \mathrm{kN}$ & 34,20 & 4,80 \\
\hline
\end{tabular}




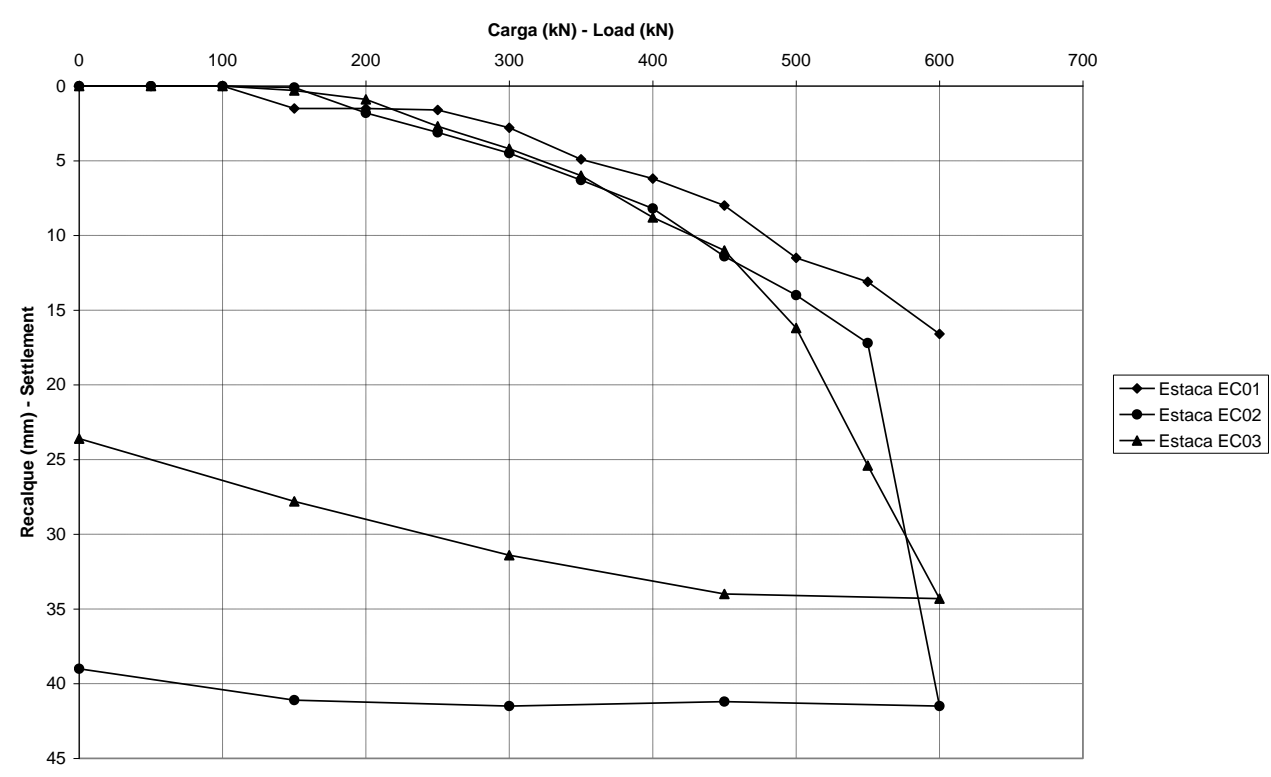

FIGURA 3. Curvas de carga versus recalque. Load versus settlement curves.

São apresentados na Tabela 3 os valores das cargas máximas previstas por meio dos métodos teóricos estudados.

TABELA 3. Cargas máximas previstas por meio dos métodos teóricos estudados. Maximum loads foreseen by using the studied theoretical methods.

\begin{tabular}{lcccc}
\hline Estaca & PC & MEY $^{* * *}(1973)$ & MEY $^{* * *}(1973)$ & GRB $^{\#}$ \\
\hline Escavada-EC01 & $700 \mathrm{kN}$ & $1.063 \mathrm{kN}$ & $1.208 \mathrm{kN}$ & $981 \mathrm{kN}$ \\
Escavada EC02 & $600 \mathrm{kN}$ & $1.063 \mathrm{kN}$ & $1.208 \mathrm{kN}$ & $981 \mathrm{kN}$ \\
Escavada EC03 & $630 \mathrm{kN}$ & $1.063 \mathrm{kN}$ & $1.208 \mathrm{kN}$ & $981 \mathrm{kN}$ \\
\hline em que: PC - carga máxima estimada pelo método de VAN der VEEN $(1953) ; \mathrm{ca}-0,8 \mathrm{c} \mathrm{e} \delta=0,95 \varnothing ;{ }^{* *} \mathrm{ca}=\mathrm{c} \mathrm{e} \delta=\varnothing ;{ }^{\#}=0 ;{ }^{\# \#=-\varnothing / 8 .}$
\end{tabular}

Na Tabela 4, são apresentadas as relações entre as cargas máximas previstas por meio dos métodos (PU) e as cargas máximas obtidas por meio das provas de carga (PC).

TABELA 4. Valores de PU/PC obtidos. Obtained values of PU/PC.

\begin{tabular}{lcccc}
\hline Estaca & $\mathrm{PC}(\mathrm{kN})$ & $\mathrm{MEY}^{* *}(1973)$ & $\mathrm{MEY}^{* *}(1973)$ & $\mathrm{GRB}^{\#}$ \\
\hline Escavada EC01 & 700 & 1,52 & 1,72 & 1,40 \\
Escavada EC02 & 600 & 1,77 & 2,00 & 1,64 \\
Escavada EC03 & 630 & 1,68 & 1,92 & 1,56 \\
\hline
\end{tabular}

em que: ca $=0,8 \mathrm{c}$ e $\delta=0,95 \varnothing ; *^{* *}$ ca $=$ c e $\delta=\varnothing ;{ }^{\#} \lambda=0 ;{ }^{\# \#} \lambda=-\varnothing / 8$.

Observando-se a Tabela 4, verifica-se que o valor de PU/PC médio para a estaca, considerando todos os métodos estudados, foi igual a 1,7 (desvio-padrão $=0,20$, coeficiente de variação $=11,2 \%$, e valores máximos e mínimos iguais a 2,0 e 1,40, respectivamente).

$\mathrm{Na}$ Tabela 5, são apresentadas as resistências laterais (RL) previstas por meio dos métodos semiempíricos estudados. São apresentadas na Tabela 6 as relações entre as resistências laterais previstas (RL) e as cargas máximas (PC) obtidas pelas provas de carga. 
TABELA 5. Resistências laterais previstas pelos métodos de compressão (RL). Lateral shear strength foreseen by the considered methods.

\begin{tabular}{lcccc}
\hline Estaca & PC & D\&Q $(1998)$ & A\&V (1975) & A\&V (1975)** \\
\hline Escavada EC01 & $700 \mathrm{kN}$ & $269 \mathrm{kN}$ & $146 \mathrm{kN}$ & $203 \mathrm{kN}$ \\
Escavada EC02 & $600 \mathrm{kN}$ & $269 \mathrm{kN}$ & $146 \mathrm{kN}$ & $203 \mathrm{kN}$ \\
Escavada EC03 & $630 \mathrm{kN}$ & $269 \mathrm{kN}$ & $146 \mathrm{kN}$ & $203 \mathrm{kN}$ \\
\hline
\end{tabular}

em que: *SPT; **CPT.

TABELA 6. Valores de RL/PC obtidos. Obtained values of RL/PC.

\begin{tabular}{lcccc}
\hline Estaca & PC $(\mathrm{kN})$ & D\&Q $(1998)$ & A\&V (1975) & A\&V (1975)** \\
\hline Escavada EC01 & 700 & 0,38 & 0,21 & 0,30 \\
Escavada EC02 & 600 & 0,45 & 0,24 & 0,34 \\
Escavada EC03 & 630 & 0,43 & 0,23 & 0,32 \\
\hline
\end{tabular}

Onde: *SPT; **CPT.

De acordo com a Tabela 6, o valor de RL/PC médio (considerando-se todos os métodos utilizados) para as estacas estudadas foi igual a 0,32 (desvio-padrão $=0,1$, coeficiente de variação $=26,7 \%$ e valores máximos e mínimos iguais a 0,45 e 0,21 , respectivamente). Deve-se notar também que o método de AOKI \& VELLOSO (1975), utilizando dados de SPT, foi o que apresentou valores médios de RL/PC mais distantes da unidade. Caso estes valores sejam retirados da média, o valor de RL/PC ficaria igual a 0,37 (desvio-padrão $=0,06$, coeficiente de variação $=16,4 \%$ ).

Na Tabela 7, são apresentadas as cargas médias (considerando todos os métodos estudados) previstas por meio dos métodos teóricos e semiempíricos utilizados.

TABELA 7. Carga média prevista (PU - métodos teóricos; RL - métodos empíricos) considerando-se todos os métodos estudados. Average load foreseen (PU-theoretical methods; RL-semi empirical methods) by the studied methods.

\begin{tabular}{lccccc}
\hline Estaca & $\mathrm{L}(\mathrm{m})$ & $\mathrm{PU}(\mathrm{kN})$ & $\mathrm{sd} / \mathrm{C} . \mathrm{V}$. & $\mathrm{RL}(\mathrm{kN})$ & sd/C.V. \\
\hline Escavada & 12 & 1.084 & $94 / 8,7 \%$ & 206 & $61,6 / 30 \%$ \\
\hline
\end{tabular}

Por meio da Tabela 7, pode ser observado que os métodos teóricos utilizados neste trabalho forneceram cargas máximas previstas superiores àquelas obtidas pelas provas de carga. Os métodos semiempíricos estimaram cargas máximas médias abaixo das determinadas pelas provas de carga. Entretanto, deve-se salientar que os métodos teóricos apresentaram menores coeficientes de variação em relação aos métodos semiempíricos, indicando, dessa forma, menor variabilidade em relação aos valores previstos.

\section{CONCLUSÕES}

Dentre todos os métodos estudados, aquele que apresentou a relação carga prevista/carga obtida no ensaio (RL/PC) mais próximo da unidade foi o de DÉCOURT \& QUARESMA (1998).

Para as estacas estudadas, os métodos teóricos apresentaram cargas máximas superestimadas em relação às obtidas pelas provas de carga. Entretanto, estes apresentaram menor coeficiente de variação.

Os métodos teóricos dependem de parâmetros de difícil obtenção e alto grau de variabilidade, como adesão estaca-solo $(\mathrm{Ca})$ e ângulo de atrito estaca-solo $(\delta)$, o que torna estes métodos de difícil utilização. Ao se admitirem valores predeterminados para parâmetros como adesão $\mathrm{Ca}$ ou $\delta$ baseado em recomendações diversas, poder-se-á estar incorrendo em erro, pois nem sempre as condições de estudo representam as condições para as quais foram obtidas as recomendações. 
Os métodos semiempíricos estudados apresentaram valores de resistência lateral mais conservadores comparando-se com os valores obtidos pelas provas de carga e por meio dos métodos teóricos.

O método de VAN der VEEN (1953) apresentou-se aplicável para as estacas estudadas, pois apresentou valores próximos àqueles apresentados pelas provas de carga.

\section{REFERÊNCIAS}

ABEF. ASSOCIAÇÃO BRASILEIRA DE EMPRESAS DE ENGENHARIA DE FUNDAÇÕES E GEOTECNIA. Manual de Especificações e Procedimentos. São Paulo: Ed. PINI, 1999. 282 p.

ABNT. ASSOCIAÇÃO BRASILEIRA DE NORMAS TÉCNICAS. NBR 12.131/91: Prova de carga estática. Rio de Janeiro, 1991. 4 p.

ALBUQUERQUE, P.J.R. Estacas escavadas, hélice-contínua e ômega: estudo do comportamento à compressão em solo residual de diabásio através de provas de carga instrumentadas em profundidade. 2001272 f. Tese (Doutorado em Geotecnia) - Escola Politécnica, Universidade de São Paulo, São Paulo, 2001.

AOKI, N.; VELLOSO, D.A. An aproximate method to estimate the bearing capacity of piles. In: CONGRESSO PANAMERICANO DE MECÂNICA DOS SOLOS E ENGENHARIA DE FUNDAÇÕES, 5., 1975, Buenos Aires. Anais... p.367-376.

BIERREZ, J.; BARRAUD, Y. Adaption dês foundations de pilones au terrain par lês méthodes de la méchanic dês sols. Paris: C.I.G.R.E, 1968. (Report 22-06).

DÉCOURT, L.; QUARESMA, A.R. Capacidade de carga de estacas a partir de valores de SPT. In: CONGRESSO BRASILEIRO DE MECÂNICA DOS SOLOS E ENGENHARIA DE FUNDAÇÕES, 4., 1998, Rio de Janeiro. Anais... v.1, p.45-54.

MARTIN, D. Calcul dés pieux et foundations a dale des pilônes de transport d'énergie électrique etude théorique et resultants d'essais em laboratoire et in situ. Paris: L'Instituit Technique du Batiment et des Travaux Publics, 1973. Suppl. 307-8.

MARTIN, D. Étude a la rupture de different ancrages sollicitées verticalement. 1966. These (Docteur-Ingénieur) - Faculte dês Sciences de Grenoble, Grenoble, 1966.

MEYERHOFF, G.G. Uplift resistance of inclined anchors and piles. In: INTERNATIONAL CONFERENCE ON SOIL MECHANICS AND FOUNDATION ENGINEERING, 8., 1973, Moscou. Proceedings... v.2, p.167-172.

PASCHOALIN FILHO, J.A. Estudo do comportamento a tração de diferentes tipos de estacas em solo de diabásio da região de Campinas - SP. 2008. 420 f .Tese (Doutorado) - Faculdade de Engenharia Agrícola, Universidade Estadual de Campinas, Campinas, 2008.

PASCHOALIN FILHO, J.A.; PEREIRA, M.; CARVALHO, D.; ALBUQUERQUE, P.J.R.; NOGUEIRA, R.C.R. Comportamento à tração de estacas hélice-contínuas executadas em solo de diabásio. In: CONGRESSO BRASILEIRO DE MECÂNICA DOS SOLOS E ENGENHARIA GEOTÉCNICA, 13., 2006, Curitiba. Anais... São Paulo: ABMS, 2006. 1 CD-ROM.

PASCHOALIN FILHO, J.A.; PEREIRA, M.; CARVALHO, D.; ALBUQUERQUE, P.J.R.; NOGUEIRA, R.C.R. Comportamento à tração de estacas tipo raiz executadas em solo de diabásio, típico da região de Campinas, Brasil. In: CONGRESSO LUSO-BRASILEIRO DE GEOTECNIA, 4., 2008, Porto. Anais... 1 CD-ROM.

POTYONDY, J.G. Skin friction between various soils and construction materials. Revista Geotéchnique, Montreal, v.11, n.4, p.339-353, 1961.

VEEN, V.V. The bearing capacity of a pile. In: CONFERENCE ON SOIL MECHANICS FOUNDATION ENGINEERING, 3., 1953, Zurich. Proceedings... v.2, 1953. p.84-90. 
Este artigo sofreu alterações por solicitação do editor em Dez/2011 conforme ERRATA publicada no Volume 31 Número 6 do periódico. (http://www.scielo.br/pdf/eagri/v31n6/v31n6a21.pdf) 